\title{
DIAGNÓSTICO OFTALMOLÓGICO DE UN CASO DE TELANGIECTASIA HEMORRÁGICA HEREDITARIA O ENFERMEDAD DE RENDU-OSLER-WEBER
}

\section{OPHTHALMOLOGIC DIAGNOSIS OF HEREDITARY HEMORRHAGIC TELANGIECTASIA OR RENDU-OSLER-WEBER DISEASE}

\author{
PIZZAMIGLIO-MARTÍN C¹, GIL-CAZORLA R², GUZMÁN-BLÁZQUEZ J³
}

\section{RESUMEN}

Caso clínico: Mujer de 45 años con episodios recurrentes de epífora hemática espontánea. La paciente presentaba epistaxis de repetición no filiadas y, tenía antecedentes familiares de hemorragia digestiva, además una de sus hijas sufría de epistaxis.

Discusión: El diagnóstico de telangiectasia hemorrágica hereditaria por oftalmólogos es un hecho infrecuente. La presencia de lágrimas con sangre o epífora hemática espontánea, en un paciente con historia de hemorragias de repetición en forma de epistaxis o hemorragia digestiva, nos tiene que hacer sospechar y buscar la presencia de esta enfermedad. La historia y exploración clínica son fundamentales en el diagnóstico de dicha enfermedad.

Palabras clave: Telangiectasia hemorrágica hereditaria, enfermedad de Rendu-Osler-Weber, hemorragia, epífora hemática.
ABSTRACT

Case report: Our patient was a 45-year-old woman who had recurrent episodes of hematic epiphora, repeated epistaxes for which no cause was found and a family history of gastric hemorrhage. One of her daughters also suffered from spontaneous hemorrhages.

Discussion: Hereditary hemorrhagic telangiectasia is rarely diagnosed by an ophthalmologist; however the occurrence of bloody tears occurring spontaneously in a patient with epistaxis or gastric hemorrhage should lead to suspicion of hereditary hemorrhagic telangiectasia or Rendu-Osler-Weber disease. It should never be forgotten that clinical examination and appropriate investigations are basic components of disease diagnosis (Arch Soc Esp Oftalmol 2008; 83: 381-384).

Key words: Hereditary hemorrhagic telangiectasia, Rendu-Osler-Weber disease, hemorrhage, hematic epiphora.

\section{INTRODUCCIÓN}

La telangiectasia hemorrágica hereditaria (THH) o enfermedad de Rendu-Osler-Weber, es una enfermedad hereditaria autosómica dominante caracteri- zada por anomalías en uno o dos genes (cromosomas 9q33-34 y 12q13) implicados en la reparación de la pared vascular. Las telangiectasias son dilataciones vasculares capilares que sangran espontáneamente o ante mínimo traumatismo.

\footnotetext{
Recibido: 8/3/07. Aceptado: 13/5/08.

${ }^{1}$ Licenciada en Medicina. Complejo Hospitalario de Toledo. Hospital Virgen de la Salud. Toledo. España.

2 Diplomada en Óptica-Optometría. Vissum Corporación Oftalmológica de Madrid. Madrid. España.

3 Doctor en Medicina. Complejo Hospitalario de Toledo. Hospital Virgen de la Salud. Toledo. España.
} 
Existen diferentes tipos de telangiectasias atendiendo a su morfología, las lineales y ramificadas (tabla I). La localización de las telangiectasias puede orientarnos a conocer la patología subyacente, así las telangiectasias periungueales son patognomónicas de lupus eritematoso sistémico, esclerodermia y dermatomiositis.

Las telangiectasias pueden aparecen en cualquier zona de la piel y mucosas, siendo muy frecuentes en los labios, lengua, cara y palma de la mano. Aparecen en la infancia aumentando de tamaño en la adolescencia y edad adulta. En ocasiones también hay pequeñas dilataciones vasculares en el tubo digestivo, aparato genitourinario y sistema nervioso central pudiendo erosionarse y sangrar.

Una forma especial de telangiectasia por su distribución y patrón hereditario es la THH. Estas lesiones vasculares aparecen de forma típica en mucosas nasal, bucal y conjuntival, en la cara, y partes de distales de extremidades como son los lechos ungueales. La clínica viene determinada por cuadros hemorrágicos como epistaxis y hemorragias digestivas. Morfológicamente las telangiectasias son radiadas y constituyen auténticas malformaciones arteriovenosas en las mucosas.

\section{CASO CLÍNICO}

Paciente de 45 años que acude a consulta por episodios recurrentes «sangrado ocular», la paciente comenta «que llora sangre». Los episodios ceden espontáneamente sin necesidad de tratamiento alguno.

La historia clínica de la paciente revelaba la existencia de epistaxis de repetición sin estudio otorrinolaringológico. No existían otros antecedentes

Tabla I. Clasificación de las telangiectasias personales sistémicos ni oftalmológicos de interés. Al profundizar en la historia clínica de la paciente, relata que su padre sufría de hemorragias digestivas altas de repetición y, que el fallecimiento de éste se produjo como consecuencia de un fenómeno hemorrágico masivo y shock hipovolémico. Además, la paciente tenía dos hijas de las que una presentaba epistaxis de repetición.

El examen biomicroscópico de la conjuntiva subtarsal tanto superior como inferior evidenciaba la existencia de lesiones telangiectásicas aisladas (figs. 1-3). Una de las telangiectasias mostraba signos de coagulación reciente en relación con fenómeno hemorrágico asociado (fig. 4). La exploración funduscópica no mostraba alteraciones en el árbol vascular, no objetivándose alteraciones del calibre de los vasos arteriales ni venosos, sin fenómenos hemorrágicos, sin exudados ni neovascularización asociadas.

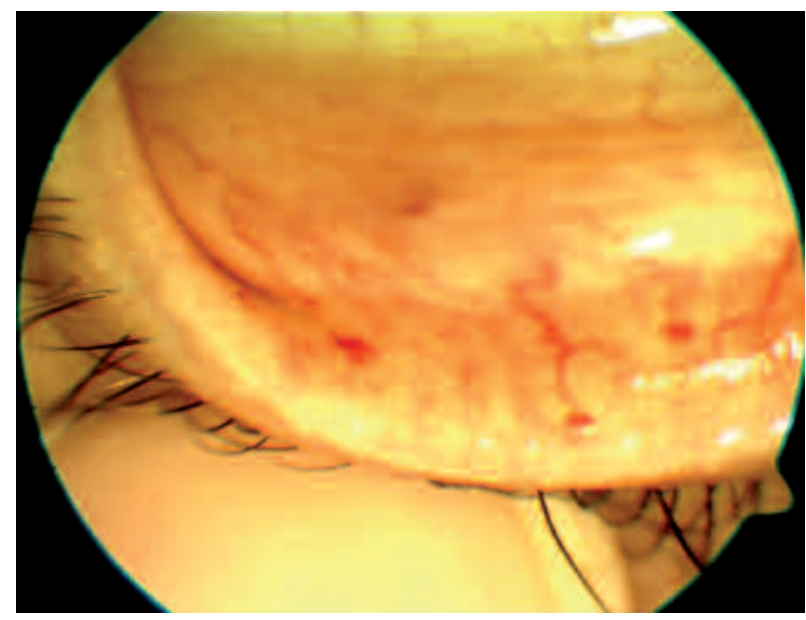

Fig. 1: Telangiectasias subtarsales inferiores $O D$.

\section{PRIMARIAS O CONGÉNITAS}

Telangiectasias esenciales generalizadas

Telangiectasia hemorrágica hereditaria de Rendu-Osler-Weber Ataxia telangiectasia de Louis-Bar

Eritema telangiectásico congénito o síndrome de Bloom

Arañas vasculares o spiders

\section{SECUNDARIAS O ADQUIRIDAS}

\section{Post-traumáticas}

Por agentes físicos: luz solar, radiaciones y frío (síndrome de Raynaud)

Fármacos: corticoides fluorados tópicos y anticonceptivos orales Dermopatías: Rosácea, Morfea, Poiquilodermia y Xeroderma pigmentoso.

Enfermedades sistémicas: Dermatomiositis, Lupus eritematoso sistémico, Esclerodermia, síndrome de CREST, síndrome carcinoide, Mastocitosis sistémica (telangiectasia macular eruptiva perstans)

Embarazo

Cirrosis hepática

Varices y síndrome postflebítico 


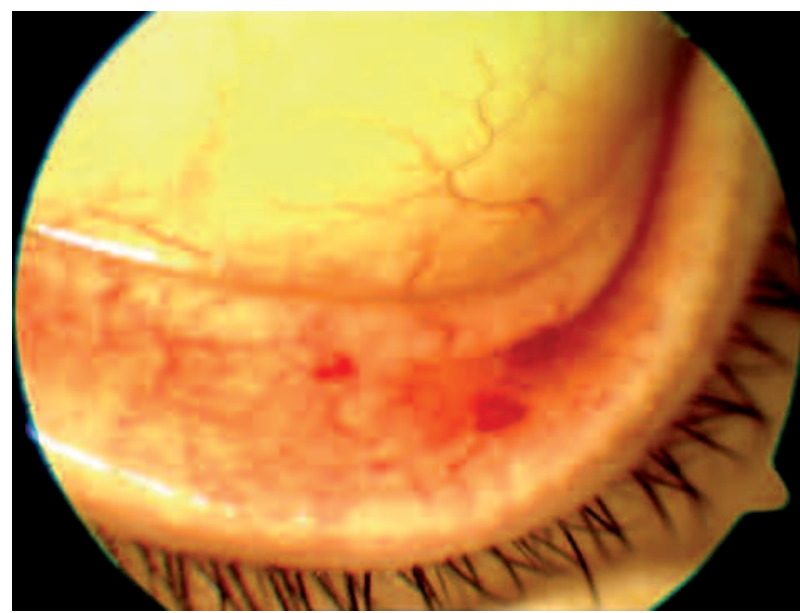

Fig. 2: Telangiectasias subtarsales inferiores OI.

El examen general de la paciente evidenciaba telangiectasias en mucosa bucal y nasal, además de lesiones telangiectásicas en la lengua y en cara bucal de labio superior e inferior (figs. 5-7).

La interconsulta al servicio de otorrinolaringología y medicina interna confirmó la presencia de telangiectasias en mucosa nasal y faríngea $\mathrm{y}$, no existiendo en la mucosa digestiva. Con todos los datos se procedió al diagnóstico de enfermedad de Rendu-Osler-Weber.

\section{DISCUSIÓN}

La THH fue descrita a finales del siglo XIX en grupos familiares caracterizados por episodios

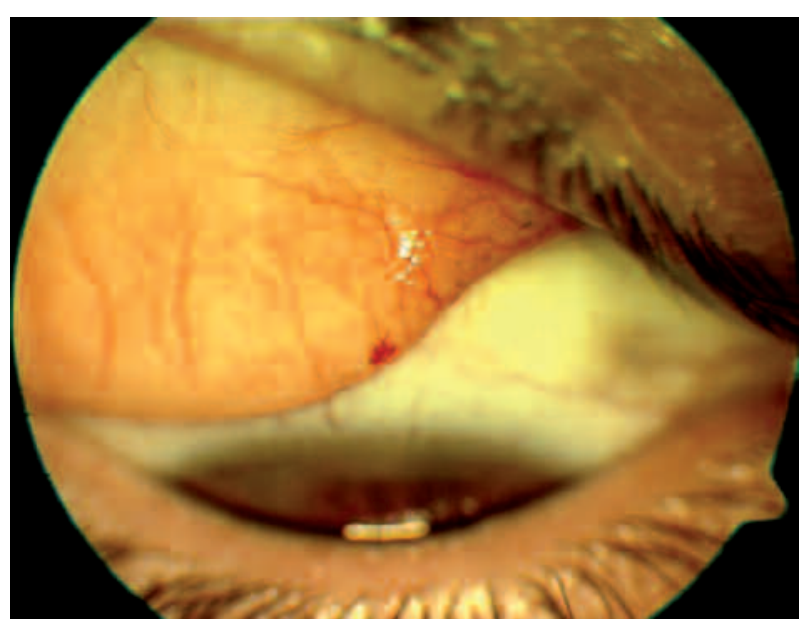

Fig. 3: Telangiectasia subtarsal superior aislada OD.

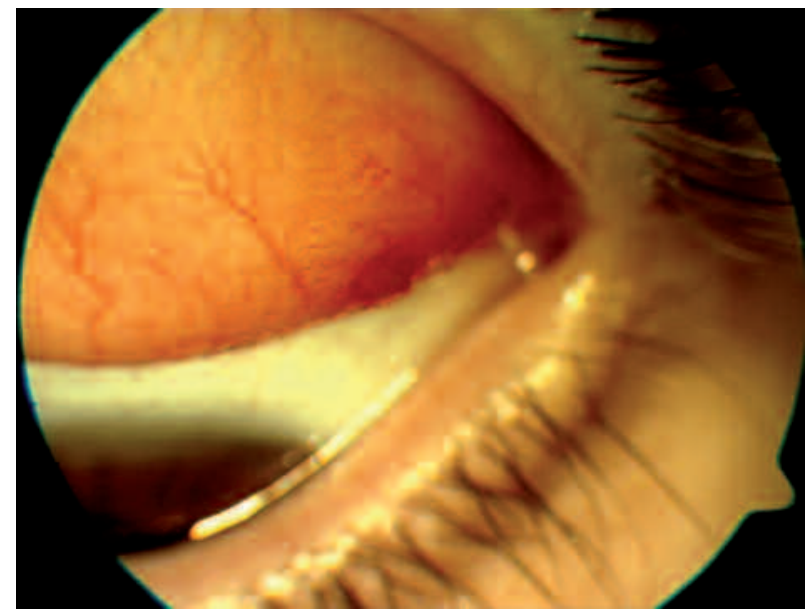

Fig. 4: Telangiectasia subtarsal superior con signos de hemorragia reciente.

espontáneos de epistaxis, hemorragia digestiva, hemoptisis o hematuria. Dichos fenómenos fueron reportados de forma independiente por tres médicos Rendu, Osler y Weber. Es Hanes (1) en 1909, quien establece la triada diagnóstica de la telangiectasia

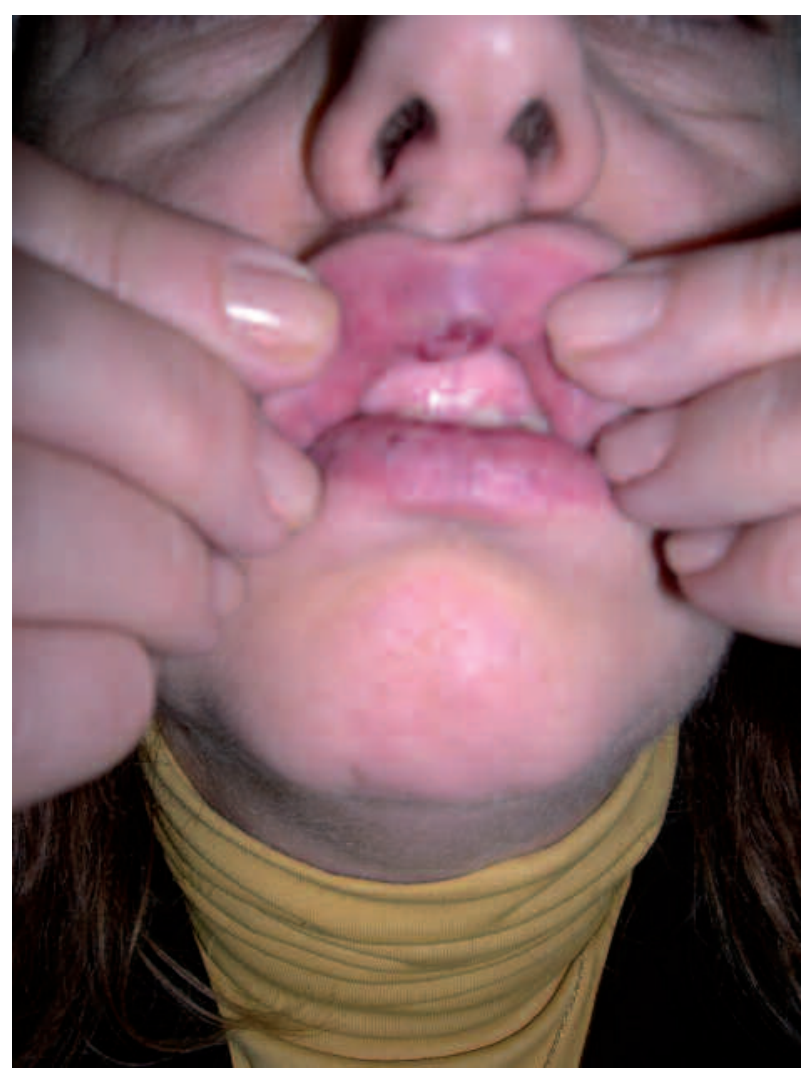

Fig. 5: Telangiectasias en mucosa labial superior. 


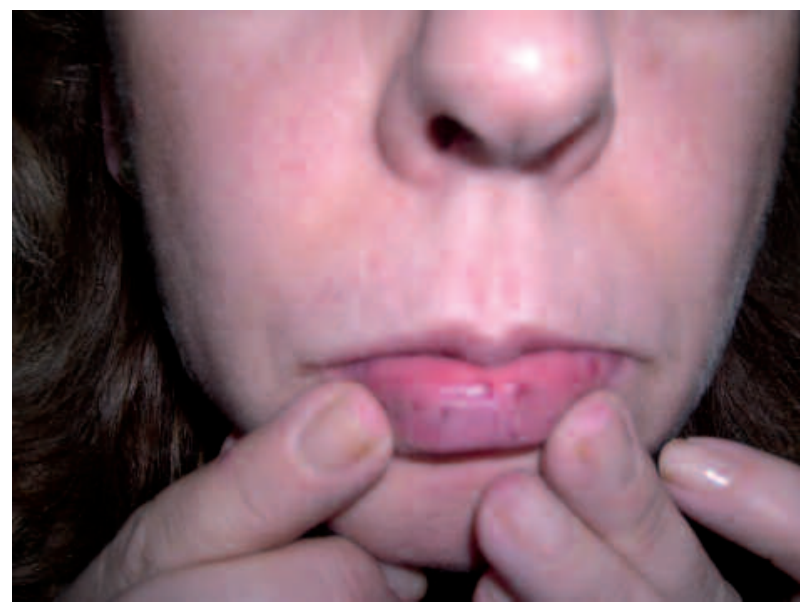

Fig. 6: Telangiectasias en mucosa labial inferior.

hemorrágica hereditaria, por asociación de historia familiar, hemorragias y telangiectasias.

La afectación ocular es un hecho infrecuente (2) y, rara vez constituye el pilar del diagnóstico (3), como es el caso que nos ocupa. Los signos y síntomas oculares se pueden agrupar en dos. Por un lado, tenemos aquellos localizados en el segmento anterior, como son lágrimas con sangre, telangiectasias y lesiones granulomatosas conjuntivales posthemorrágicas. Por otro lado, las telangiectasias retinianas maculares y coroideas (4).

El diagnóstico de presunción se hace por la clínica del paciente. El examen clínico requiere el estudio endoscópico de la mucosa digestiva y del área otorrinolaringológica. En nuestro caso, al acudir la paciente en primera instancia a nuestra consulta, se realizó una inspección de la cavidad bucal y nasal, siendo remitida con posterioridad a los servicios de otorrinolaringología y medicina interna para completar el diagnóstico y, hacer el seguimiento correspondiente.

Este caso nos muestra, como una enfermedad con implicaciones vitales por los fenómenos hemorrágicos espontáneos masivos, puede pasar desapercibida tanto para el paciente como para el médico no entrenado.

La afectación conjuntival rara vez requiere tratamiento dado la autolimitación espontánea del mismo. Se ha utilizado como terapia en algunos casos la cauterización de las telangiectasias (3).

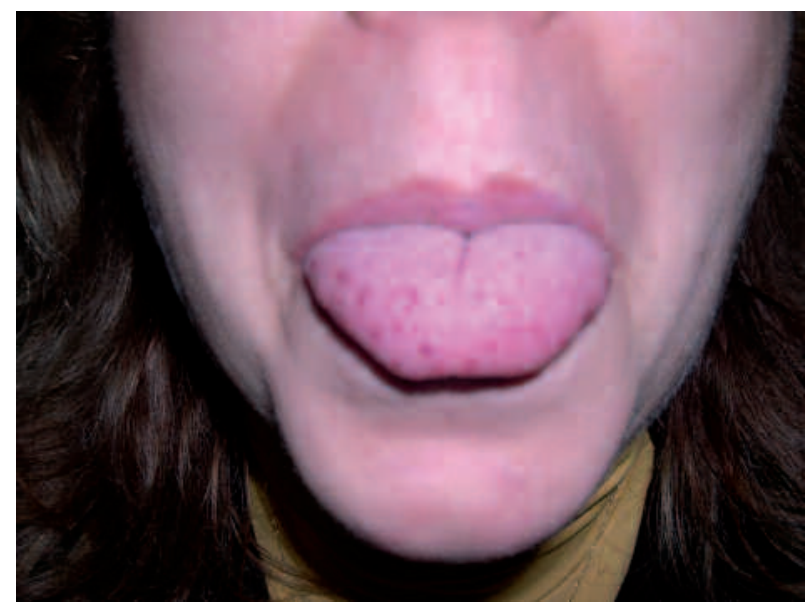

Fig. 7: Telangiectasias en lengua.

En conclusión, la existencia de fenómenos hemorrágicos oculares espontáneos en ausencia de factores etiológicos responsables (5) (hemofilia, histeria, conjuntivitis, tumores vasculares, traumatismos, granulomas, tratamiento con nitrato de plata y, regurgitación hemática conducto lacrimal) nos tiene que poner sobre aviso de la posible existencia de anomalías vasculares y, realizar una historia clínica sistémica precisa en busca de fenómenos hemorrágicos de iguales características en otras estructuras del organismo.

\section{BIBLIOGRAFÍA}

1. Guttmacher AE, Marchuk DA, White RI Jr. Hereditary hemorrhagic telangiectasia. N Engl J Med 1995; 333: 918-924.

2. Brandt AM, Schachat AP, White RI. Ocular manifestations in hereditary hemorrhagic telangiectasia (Rendu-OslerWeber disease). Am J Ophthalmol 1989; 107: 642-646.

3. Soong HK, Pollock DA. Hereditary hemorrhagic telangiectasia diagnosed by the ophthalmologist. Cornea 2000; 19: 849-850.

4. Tsai DC, Wang AG, Lee AF, Hsu WM, Liu JH, Yen MY. Choroidal telangiectasia in a patient with hereditary hemorrhagic telangiectasia. Eye 2000; 16: 92-94.

5. Wilhelmus KR, Huang AJ, Huang DG, Parrish CM, Sutphin JE, Whitsett JC. Clinical aspects of ocular surface disorders. In: External disease and Cornea. Basic and Clinical Science Course. Section 8. Chapter VI. The Foundation of the American Academy of Ophthalmology; 2001; 78. 